\title{
LIN-39/Hox triggers cell division and represses EFF-1/fusogen-dependent vulval cell fusion
}

\section{Gidi Shemer and Benjamin Podbilewicz ${ }^{1}$}

Department of Biology, Technion-Israel Institute of Technology, Haifa, 32000, Israel

General mechanisms by which Hox genes establish cell fates are known. However, a few Hox effectors mediating cell behaviors have been identified. Here we found the first effector of LIN-39/HoxD4/Dfd in Caenorhabditis elegans. In specific vulval precursor cells (VPCs), LIN-39 represses early and late expression of EFF-1, a membrane protein essential for cell fusion. Repression of $e f f-1$ is also achieved by the activity of CEH-20/Exd/Pbx, a known cofactor of Hox proteins. Unfused VPCs in lin39(-);eff-1(-) double mutants fail to divide but migrate, executing vulval fates. Thus, lin-39 is essential for inhibition of EFF-1-dependent cell fusion and stimulation of cell proliferation during vulva formation.

Supplemental material is available at http://www. genesdev.org.

Received October 4, 2002; revised version accepted October $15,2002$.

Throughout animal evolution, members of the Hox family of homeodomain proteins have been responsible for establishing regional identities along the anterior-posterior axis. Much has been learned about Hox genes, in particular from their expression patterns, and about downstream genes that are targets of Hox transcriptional regulation. Although most of the downstream targets identified thus far encode for transcription factors or signaling molecules, only a very limited number of Hox target genes have been found to be effectors or genes encoding structural proteins that participate in the actual intra- and intercellular events during morphogenesis (Graba et al. 1997). A given Hox gene can function in different cell types and at multiple times during development, activating some targets and inhibiting others (Salser and Kenyon 1996; Graba et al. 1997). Part of this differential function depends on the presence or absence of proteins (e.g., EXD/PBX) that work as coactivators or corepressors along with the Hox genes (Mann and Affolter 1998). The Hox gene lin-39, the paralog of deformed $(D f d)$ from Drosophila and mammalian HoxD4, controls vulva formation as well as other developmental events in Caenorhabditis elegans (Clark et al. 1993;

[Keywords: Caenorhabditis elegans; cell fusion; vulva development; Hox]

${ }^{1}$ Corresponding author.

E-MAIL podbilew@tx.technion.ac.il; FAX 972-4-822-5153.

Article and publication are at http://www.genesdev.org/cgi/doi/10.1101/ $\operatorname{gad} 251202$.
Wang et al. 1993; Eisenmann et al. 1998; Grant et al. 2000; Liu and Fire 2000). In one case, lin-39 was shown to act with ceh-20, an ortholog of the extradenticle (exd) cofactor, to regulate patterning of the postembryonic mesoderm in C. elegans (Liu and Fire 2000).

During vulva development, lin-39 possibly acts at two developmental stages. At the first larval stage (L1), it is active in six of eleven ventral epidermal Pn.p cells, inhibiting them from fusing to the surrounding hypodermis (epidermis) like their sister cells (Ch'ng and Kenyon 1999). At the third larval stage (L3), lin-39 permits three of these vulval precursor cells [VPCs; $\mathrm{P}(5-7) . \mathrm{p}$ ], to escape the fusion fate (Maloof and Kenyon 1998; Gleason et al. 2002). Although expressed at low levels in the other three VPCs $[\mathrm{P}(3,4,8) . \mathrm{p}]$, lin-39 is not sufficient for these cells to escape cell fusion (Clandinin et al. 1997; Maloof and Kenyon 1998). Only when its activity is elevated (e.g., through ectopic Wnt signaling) can these cells escape fusion and adopt vulval fates (Fig. 1; Gleason et al. 2002).

Due to its importance as a Hox/homeotic gene, and specifically as the most downstream gene in the signaling pathways involved in vulva formation, lin-39 was intensively studied in the last decade. It was found that at the first larval stage, its expression is suppressed in the posterior body region by the chromatin regulatory factor egl-27 (Ch'ng and Kenyon 1999) as well as by redundant activity of the hairy homolog ref-1 and the Hox gene mab-5 (Alper and Kenyon 2001). In males, the zinc-finger protein REF-2 also interacts with lin-39 and other Hox genes to regulate cell fusion in Pn.p cells (Alper and Kenyon 2002). At L3, lin-39 expression is elevated due to activity of the transcription factor sem-4 (Grant et al. 2000) and members of the Wnt pathway (Eisenmann et al. 1998; Hoier et al. 2000). However, how lin-39 regulates cell fusion and whether it has other roles in patterning vulva formation remain unknown.

Whereas in C. elegans and related nematodes, the nonvulval Pn.p cells fuse with the hypodermis (LouvetVallee et al. 2002), the equivalent cells in Pristionchus pacificus (a nematode that has been separated from $C$. elegans for $\sim 100$ million years) undergo programmed cell death (Felix 1999; Shemer and Podbilewicz 2000). This apoptotic fate is inhibited in the VPCs by the activity of the $P$. pacificus lin-39, probably by repression of the apoptotic effector ced-3, the only candidate effector regulated by a $D f d$ paralog (Eizinger and Sommer 1997). These evolutionary differences between C. elegans and $P$. pacificus could be explained by differences in the regulatory sequences of the lin-39 promoter (Grandien and Sommer 2001), or due to different effectors expressed in the VPCs in each species. To expand on the finding that lin-39 inhibits cell fusion of vulval cells in C. elegans, we asked whether it does so by inhibiting a fusion effector. We recently isolated eff-1, a gene encoding type-I membrane proteins essential for all epithelial cell fusion events throughout embryonic and postembryonic development (Mohler et al. 2002). Here, we asked whether eff- 1 may be a cell fusion effector down-regulated by lin39 and other cofactors and whether lin-39 acts only permissively to inhibit cell fusion of specific Pn.p cells or also to execute vulva formation through other effectors of cell division and cell migration. 


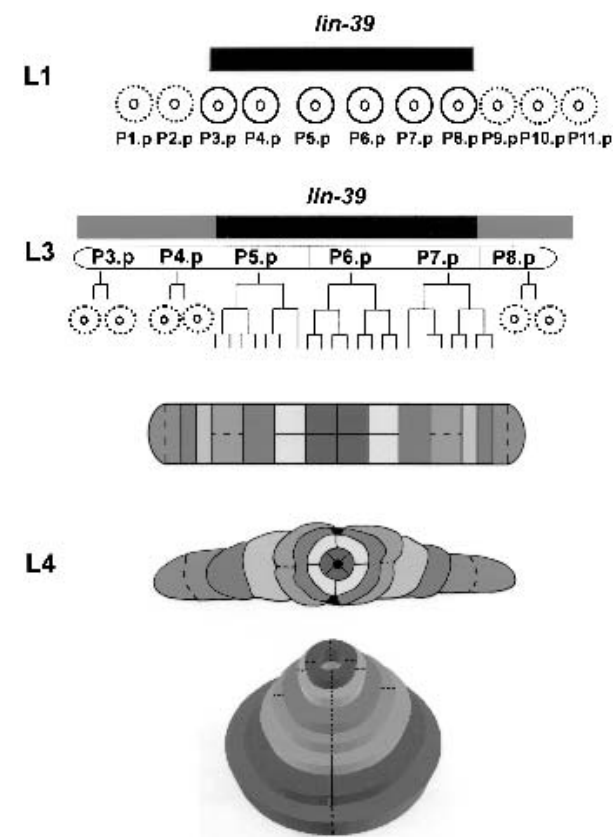

Figure 1. lin-39 inhibits cell fusion during vulva formation. lin-39 is expressed and active at the first larval stage (L1) in six epidermal cells (P3.p-P8.p; black circles), also known as the vulva precursor cells (VPCs). This activity (black box above cells) is necessary and sufficient to inhibit the VPCs from adopting the fate of their neighboring cells $[\mathrm{P}(1,2,9-11) \cdot \mathrm{p}]$ - fusion to the surrounding syncytium hyp7 (dashed circles). At L3, the VPCs are subjected to various signaling pathways. $\mathrm{P}(3,4,8)$.p undergo one cycle of division, followed by fusion of their daughter cells to hyp7 (dashed circles). In about half of the cases, P3.p does not divide before cell fusion. P(5-7).p escape cell fusion and continue to divide, yielding a 22-cell vulval primordium. It was proposed that basal activity of LIN-39 in $\mathrm{P}(3,4,8) \cdot \mathrm{p}$ is not sufficient for these cells to escape cell fusion, and only elevated activity of LIN-39 induced by the Ras pathway in $\mathrm{P}(5-7) . p$ in wild-type (Maloof and Kenyon 1998) or by ectopic Wnt signaling in all VPCs in some mutants (Gleason et al. 2002), allows these cells to escape cell fusion (black and shaded boxes above cells). Migration, cell fusion (dashed lines), ring formation, and invagination of the primordial cells at L4 lead to a tube-shaped adult vulva (Sharma-Kishore et al. 1999).

\section{Results and Discussion}

\section{eff- 1 is epistatic to lin-39}

In C. elegans, lin-39 may repress VPCs from cell fusion fate in the first and third larval stages (L1 and L3), may promote vulval fates by keeping VPCs responsive to other signaling events in L3, or may combine both activities, inhibiting cell fusion and activating the response to inductive signals downstream of the RAS pathway (Clandinin et al. 1997; Maloof and Kenyon 1998; Gleason et al. 2002). In the lin-39(n1760) loss-of-function null mutant, all Pn.p cells have a cell fate transformation and fuse with the hypodermis at L1, developing no vulva (Clark et al. 1993; Wang et al. 1993). In contrast, in the eff-1(hy21) mutant, none of the Pn.p cells fuse at L1 or at L3, a protruded vulva is formed, and some animals have extra vulvae (Mohler et al. 2002).

To test the genetic epistatic relationship between lin39 and eff-1, we followed the fates of the different VPCs in wild-type, single, and double mutants during the L1 and L3 developmental stages. Several signaling pathways determine distinct cell fates for descendants of the different VPCs (Sharma-Kishore et al. 1999). We found that in wild-type worms, only $\mathrm{P}(3-8)$.p cells escaped fusion in $\mathrm{L} 1$; $\mathrm{P}(5-7)$.p cells escaped fusion fate in L3, divided, and yielded the characteristic seven vulval rings at the L4 stage (Figs. 1, 2A,E). In lin-39(n1760) null mutants, all of the Pn.p cells fused to the surrounding hypodermis at mid-L1 (Fig. 2B,F). A similar pattern was seen in lin39(n1490ts) conditional mutants (data not shown): at the semipermissive temperature of $20^{\circ} \mathrm{C}$, occasionally one Pn.p cell escaped cell fusion at L1. This cell could either fuse at the L3 stage or it could stay unfused through L3. However, even in the latter case, this cell yielded an abnormal and nonfunctional "mini-vulva." In eff-1(hy21) mutants, all of the VPCs $[\mathrm{P}(3-8) \cdot \mathrm{p}]$ and the

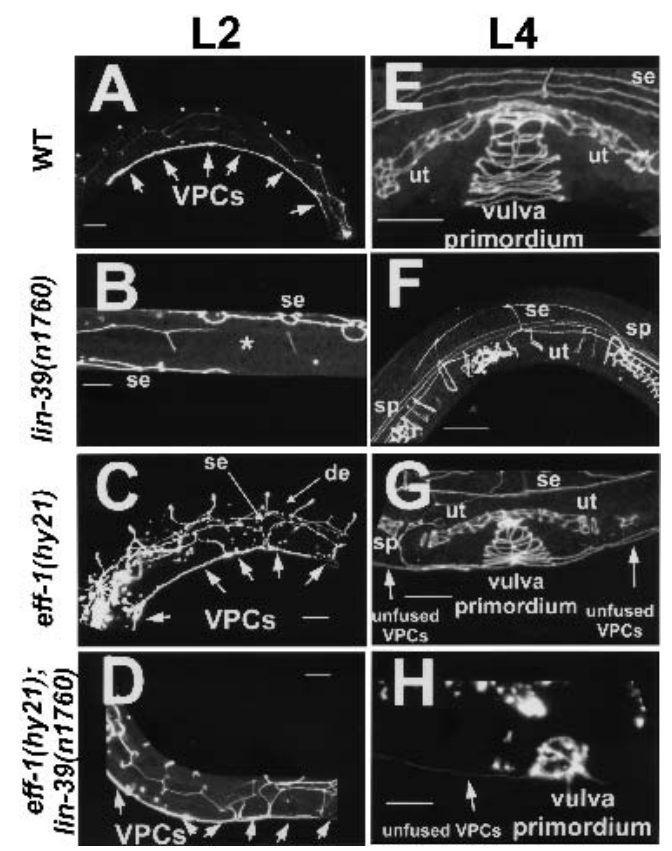

Figure 2. eff-1 is epistatic to lin-39 during early and late cell-fusion events. Confocal reconstructions of worms carrying an aim-1::GFP construct marking the adherens junctions of epithelial cells (Shemer et al. 2000; Koeppen et al. 2001). The worms were assayed at early L2 $(A-D)$ after the early fusion events of mid-L1 and at mid-L4 $(E-H)$ after the late fusion events of mid-L3 (see Fig. 1 for details). (A) Wild-type worms showing the six VPCs that escape fusion to the hypodermis after they attach to each other $(\mathrm{n}>100)$. $(E)$ Three of these VPCs escape cell fusion to hyp7 at L3, and their 22 greatgranddaughters invaginate, forming a stack of seven rings $(\mathrm{n}>100)$. $(B, F)$ In lin-39(n1760) single null mutants, no VPCs formed after all of the Pn.p cells had fused to the hypodermis ( $\left.{ }^{\star}, \mathrm{n}=50\right)$, resulting in the absence of a vulva (Vul phenotype) at late L4 $(F ; \mathrm{n}=100) .(C)$ eff-1(hy21) mutants grown at the restrictive temperature of $25^{\circ} \mathrm{C}$ showing the VPCs that fail to fuse with hyp7 (n = 50). P3.p, P4.p, and P8.p also fail to fuse in the L3 and attach to the vulva primordium formed from the descendants of $\mathrm{P}(5-7)$.p. $(G)$ The result is a stack of rings connected to a row of ectopic cells $(\mathrm{n}=70)$. Due to fusion failure in eff-1(hy21), ectopic dorsal epithelia (de) and lateral hypodermal seam cells (se) are present and migrate throughout the body of the worm. $(D, H)$ Pn.p cells fail to fuse in eff-1(hy21);lin-39(n1760) double mutants at $\mathrm{L} 1(D ; \mathrm{n}=45)$ and later at L3 $(H ; \mathrm{n}=28)$. The unfused VPCs migrate and invaginate, forming a "pseudo" vulval primordium that is incomplete and abnormal structurally, resulting in a nonfunctional vulva (cf. $H$ and $G, E$ ). ut, uterus; sp, spermatheca. Anterior is to the left and dorsal is up, except for $B$, a ventral view. The fusion status of the cells was also confirmed by staining worms with the MH27 antibody (Podbilewicz and White 1994). Arrows mark unfused cells. Bar, $10 \mu \mathrm{m}$. 
$\mathrm{P}(1,2,9-11) . p$ cells escaped fusion at $\mathrm{L} 1$ and failed to fuse again during the L3 stage, forming a vulva primordium of unfused vulval cells linked to unfused epidermal cells (Fig. 2C,G). If the double mutant had the phenotype of a single eff-1, with respect to the fusion of the Pn.p cells, it would mean that lin-39 controls cell fusion in C. elegans by inhibiting the activity of eff-1. We found that in lin39(n1760);eff-1(hy21) double mutants, all of the Pn.p cells failed to fuse both at L1 and at L3 despite the fact that lin-39 inhibitory activity was absent (Fig. 2D,H). Similarly, the use of a lin-39(n1490ts) weaker allele in combination with eff-1(hy21ts) showed the same phenotype. Thus, during cell fusion events regulated by lin-39 in vulva formation, eff-1 is epistatic to lin-39. This is true for the VPCs at L1 where lin-39 is active, and for $\mathrm{P}(5-7) . p$ at L3 where there is sufficient lin-39 activity to maintain these cells unfused. The similarity of the cell fusion phenotypes of eff-1 single mutants and lin-39; eff-1 double mutants suggests that eff- 1 acts downstream of lin-39 at both early and late fusion events during vulva formation.

\section{lin-39 represses eff-1 expression}

To rule out an alternative model in which lin-39 inhibits cell fusion in the VPCs by repressing an unknown fusion effector that works nonredundantly with eff-1 to promote cell fusion, we analyzed the expression of eff-1 RNA in wild-type and lin-39 mutant worms using a transcriptional eff-1p::GFP fusion construct (Mohler et al. 2002). Analysis of wild-type worms showed that eff$1 p:: G F P$ was expressed in the daughter cells of P3.p, P4.p, and P8.p that are committed to fusion, and was absent in the daughter cells of $\mathrm{P}(5-7) \cdot \mathrm{p}$ which escape the fusion fate (Fig. 3A). In contrast, in lin-39(n1760) null mutants, which completely lack LIN-39 activity, all of the VPCs expressed eff-1p::GFP (Fig. 3B). Thus, the expression of eff-1 RNA is regulated by LIN-39 activity, showing that eff-1 works downstream of lin-39 rather than in parallel. Consistent with these results, at the restrictive temperature of $25^{\circ} \mathrm{C}$, lin-39(n1490ts) mutants expressed eff-1p::GFP in all of the VPCs. After shifting these conditional mutant animals to the permissive temperature, we found that GFP expression was completely dependent on LIN-39 activity. eff-1p::GFP was absent in $\mathrm{P}(5-7) . p$ only in cases where remnants of lin-39 activity were present, permitting $\mathrm{P}(5-7) . \mathrm{p}$. to be further induced and to adopt vulval fates. In summary, our results show that lin-39 inhibits epidermal fusion of the VPCs $[\mathrm{P} / 3-$ 8).p] at L1 and that of $\mathrm{P}(5-7) \cdot \mathrm{p}$ at L3 by repressing eff-1 expression (Fig. 4A).

\section{ceh-20/exd represses eff-1-dependent fusion} and vulva expression

A previous study showed that lin-39 acts together with the exd homolog ceh-20 in C. elegans to regulate the twist transcription factor ortholog hlh-8 in postembryonic mesodermal cells (Liu and Fire 2000). To test whether ceh-20 participates in regulation of cell fusion, we analyzed the fusion pattern of the VPCs in ceh20(ay42) mutants. We found that all VPCs fused to hyp7 at mid-L3 in these mutant animals, except for one case in which one VPC escaped cell fusion. As a result, all of these mutants lacked a vulva (Fig. 3C). To test whether this fusion inhibition involves repression of eff-1, we

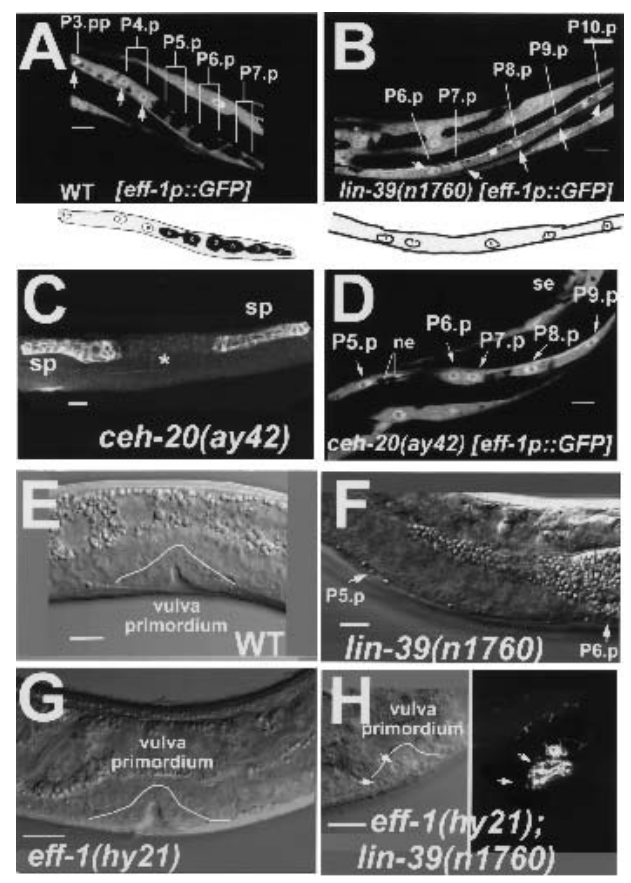

Figure 3. lin-39 and ceh-20 repress eff- 1 expression. $(A, B)$ Z-serial sections from confocal reconstructions of L3 worms showing expression of eff-1promoter::GFP (white) in vulval primordial cells in the presence $(A)$ and absence $(B)$ of lin-39 activity. $(A)$ White arrows mark nuclei of cells that fuse with the surrounding hypodermal syncytium hyp7. All of these nuclei express eff-1 (arrows and white circles in the diagram beneath; nucleoli are small circles within nuclei, $n=35)$. Daughter cells of $\mathrm{P}(5-7) \cdot \mathrm{p}$, which escape fusion to the hypodermis due to lin-39 activity, do not express eff-1. (B) In the absence of lin-39 activity, all of the VPCs express eff-1 [P(6-10).p in this picture; see arrows and white nuclei in the diagram beneath; $\mathrm{n}=40] .(C, D)$ ceh-20 inhibits cell fusion by repressing eff- 1 expression. (C) ceh-20 L4 mutant stained with MH27 showing that in the absence of ceh-20, no vulva $\left(^{\star}\right)$ is formed as a result of VPC fusion $(\mathrm{n}=18)$. The uterine cells are shown in the weak staining between the spermathecae (sp). (D) Expression of eff-1promoter::GFP (white) in vulval primordial cells in the absence of ceh-20 activity. These cells express GFP $[\mathrm{P}(5-9)$. $\mathrm{p}$ in this picture]. Seam cells (se) and ventral neural cells (ne) are also shown $(\mathrm{n}=22)$. $(E-H)$ Nomarski micrographs showing vulval nuclei during organogenesis. $(E)$ Wild-type worm at late L4. This vulva primordium is comprised of 22 nuclei (white line, invagination with 10 nuclei in this focal plane; $\mathrm{n}>100$ ). (F) lin-39(n1760) null mutant worm at early L4 showing only P5.p and P6.p that in the absence of lin-39 activity fused to hyp7 ( $\mathrm{n}=30)$. (G) eff-1(hy21) worm at exactly the same stage as that in $E$ (white line, invagination; $\mathrm{n}=60)$. $(H)$ In the absence of lin-39, the VPCs do not proliferate in eff-1(hy21);1in-39(n1760) mutants (n = 34). However, the cells that fail to fuse in the absence of eff-1 activity, migrate and ultimately invaginate (white line). Right image shows a stack of four vulval rings $(a j m-1:: G F P)$ from the same vulva. Anterior is left and dorsal is up, except for $A, C$, and $D$, which are ventral views. All worms were grown at $25^{\circ} \mathrm{C}$ for complete penetrance of the eff-1(hy21) mutation. Bar, $10 \mu \mathrm{m}$.

tested eff-1p::GFP expression in ceh-20(ay42) mutants. As in the case of lin-39(-), in the absence of ceh-20 activity, GFP expression was detected in all VPCs at L3 (Fig. 3D). Thus, ceh-20 also regulates cell fusion by repressing eff-1 expression in specific cells. Whether LIN39 acts with CEH-20 as a protein complex and whether this regulation is direct will be tested in future experiments.

In addition to the vulval ectodermal cells, eff- 1 is essential for cell fusion in many epithelial and mesodermal 


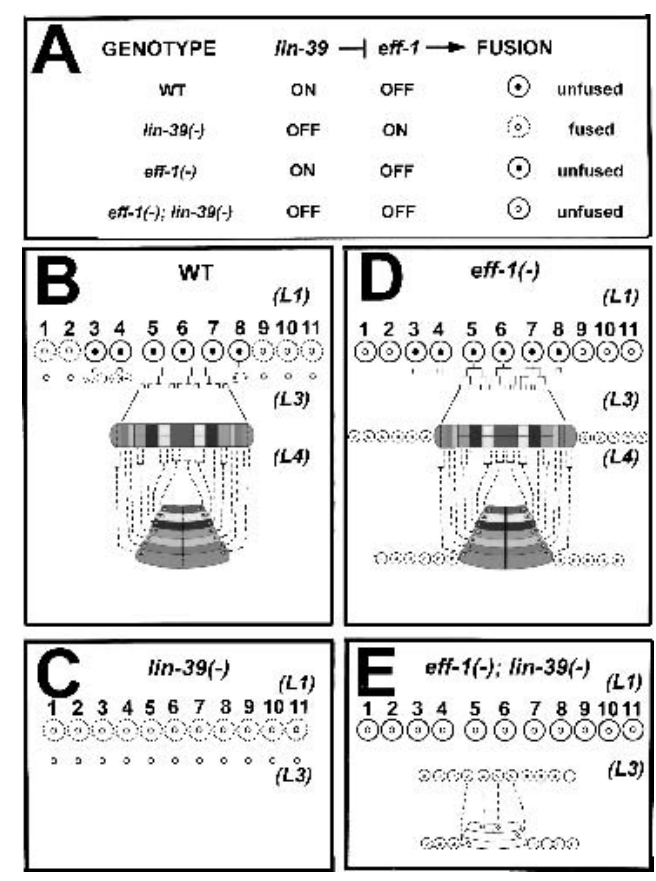

Figure 4. A model of lin-39 activity during vulva formation. (A) lin-39 inhibits cell fusion in the VPCs by repressing the fusogen eff-1. This model of interactions among lin-39, eff-1, and cell fusion predicts the fusion pattern of the VPCs in wild-type, single, and double mutants. In all diagrams, dashed lines represent fusing cells, and solid lines represent nonfusing cells. Cells that express LIN-39 have black nuclei. $(B-E)$ Structural fates of the vulval cells in different genotypes. $(B)$ In wild-type worms, lin-39 acts in $\mathrm{P}(3-8)$.p at L1 and in $\mathrm{P}(5-7) \cdot \mathrm{p}$ at $\mathrm{L} 3$, preventing these cells from fusion. The result is a 22-cell primordium that forms the seven vulval rings. $(C)$ In lin-39(-) single mutants, eff-1 is not repressed and at L1, all cells fuse and contribute their nuclei to the surrounding hypodermis. $(D)$ In eff-1(-) single mutants, none of the cells are able to fuse with the hypodermis, resulting in ectopic cells that migrate along with the vulva precursors. (E) In eff-1(-); Iin-39(-) double mutants, despite the lack of the inhibitory activity of lin-39, the Pn.p cells are not able to fuse at L1 and at L3 in the absence of eff- 1 activity. In the absence of lin-39, the cells fail to proliferate. They do succeed in forming rings (three in this example), but these rings are structurally abnormal and nonfunctional.

cells in which lin-39 is not expressed or active (Shemer and Podbilewicz 2000). Other transcription factors that are known to regulate fusion in these cells might also work by regulating eff-1 expression (Witze and Rothman 2002). Future work analyzing the relationships between eff-1 and these regulators (e.g., the ELT-5/6 GATA transcription factors in the seam cells) should give a comprehensive view on how cell fusion is controlled throughout development in C. elegans.

\section{lin-39 is required for proliferation of vulval cells}

In wild-type worms the unfused VPCs divide, migrate, form rings, and invaginate to form the adult vulva (Fig. 4B; Sharma-Kishore et al. 1999). It has been proposed, using temperature-sensitive lin-39 mutants and heatshock promoter::lin-39 transgenic worms, that the activity of lin-39 is essential to keep the VPCs responsive to other inductive signaling events (Clandinin et al. 1997; Maloof and Kenyon 1998). Because cell fusion could not be bypassed in those experiments, the fusion of the VPCs alone, in the absence of lin-39 activity, could explain lack of vulval induction (Fig. 4C). The exact role of lin-39 during late stages of vulva development is thus unclear; lin-39 may have additional effectors in the VPCs and may be responsible for one or more of three processes: maintaining inhibition of cell fusion, promoting proliferation of $\mathrm{P}(5-7) \cdot \mathrm{p}$, and/or inducing formation of vulval rings. To prevent cell fusion of the VPCs to the hypodermis, we used eff-1(hy21) at $25^{\circ} \mathrm{C}$ at the L3 stage (Figs. 3G, 4D) and investigated the behavior of these cells and whether they were able to form vulval rings in lin39(-);eff-1(-) and lin-39(ts);eff-1(-). We found that although the VPCs escaped fusion in the double mutants, they failed to proliferate (Figs. $3 \mathrm{H}, 4 \mathrm{E}$ ). These results show that (1) cells that fail to fuse because the cell fusion effector is inactive in eff-1(-) need additional cues to divide, and (2) lin-39 may control a cell division effector in the VPCs that is required for them to divide. In the double mutant, the unfused VPCs may be blocked in proliferation because a putative cell division effector of lin-39 is not receiving positive input from this Hox gene. In wild-type worms, remnants of lin-39 from previous stages may be sufficient to induce one cycle of division in $\mathrm{P}(3,4,8)$.p before fusion to epidermis, whereas in the lin-39(-);eff-1(-) mutants, residual lin-39 activity is absent, resulting in a block of cell division. Thus, in wildtype animals, lin-39 may activate an unidentified effector of cell division in the VPCs, and lin-39 complete loss-of-function causes cell cycle arrest. Alternatively, in the absence of lin-39 activity, unfused VPCs may undergo a cell fate transformation from vulval precursor cells to differentiated vulval fates equivalent to the great-granddaughters of $\mathrm{P}(5-7) \cdot \mathrm{p}$ cells.

\section{Unfused stem cells (VPCs) differentiate without dividing in lin-39(-);eff-1(-) mutants}

We next investigated the behavior of nonproliferative unfused cells. During the first hours of the L4 stage, the primordial cells normally form rings and invaginate. In lin-39(-);eff-1(-) animals, unfused and undivided VPCs did not show any morphological changes. Surprisingly however, at mid-L4 the VPCs succeeded to form one to four vulval rings or half-rings that stacked to form a pyramidal invagination (Figs. $2 \mathrm{H}, 3 \mathrm{H}, 4 \mathrm{E}$ ). Thus, lin-39 is not necessary to promote cell migration and ring formation during vulva development. These results imply that there are no cell migration effectors of lin-39 in the process of vulva formation. To further examine the role of unfused and undivided VPCs in vulva morphogenesis, we examined the formation of vulval structures and found that the vulval rings were defective compared to wild-type and eff- 1 animals (Fig. 3, cf. H and E,G), and these vulvae were nonfunctional. Actually, these structures resembled the structures of pseudovulval rings seen in let-60/ras (gf) mutants that also result from a relatively small number of precursors (Shemer et al. 2000), showing that in C. elegans, a 6-cell vulval primordium is not sufficient to form a functional organ. In conclusion, our findings show that vulval/epidermal stem cells in $C$. elegans continue a differentiation program regardless of cell division. We demonstrated that these cells in developing worms continue their cell fate determination and execution programs even when the cell-division cycle is blocked, as occurs in other organisms (Harris and Hartenstein 1991; Amthor et al. 1998). 
In summary, lin-39 activity is essential for two sequential stages in the VPCs: inhibition of eff-1-dependent cell fusion to the epidermis, and activation of celldivision cycle mediated by an unidentified Hox effector of proliferation. In addition, it appears that lin-39 does not control cell migration effectors essential for ring formation and stacking during invagination. Because other Hox genes in Drosophila, vertebrates, and C. elegans also regulate multiple cell behaviors such as migration fusion, proliferation, differentiation. and morphogenesis (Salser and Kenyon 1996), it is possible that some of the unidentified effectors are evolutionarily conserved. Identification of these effectors may facilitate the understanding of mechanisms that regulate stem cell division, fusion, and differentiation in mammals (Ying et al. 2002).

\section{Materials and methods}

\section{General methods and strains}

Worms were handled as described (Brenner 1974). All experiments were performed at $20^{\circ} \mathrm{C}$ unless otherwise indicated. Wild-type animals were $C$. elegans Bristol N2 strain. The following genes and alleles were used: (LGII) eff-1(hy21) (Mohler et al. 2002); (LGIII) lin-39(n1760), lin39(n1490ts), ceh-20(ay42), dpy-17(e164), unc-32(e189), unc-36(e251); (LGIV) him-8(e1489) (Riddle et al. 1997). SU93 jcIs1\{ajm-1::GFP pRF4[rol-6(su1006)]\}IV (Koeppen et al. 2001) served as control in all experiments testing strains on a jcIs1 background.

FC50 zzEx10[pJE3, pRF4] contains pJE3, an eff-1p::GFP transcriptional construct comprised of a 7.5- kb promoter sequence of $e f f-1$ fused to the GFP vector pPD95.75 (gift from A. Fire; Mohler et al. 2002).

To analyze single and double mutants with or without eff-1p::GFP expression, the following strains were generated: BP92 [eff-1(hy21)II; unc-36(e251), lin-39(n1490ts)III; jcIs1 IV], BP93 [unc-36(e251), lin39(n1490)III; jcIs1 IV], BP94 [eff-1(hy21)II; lin-39(n1760)III; jcIs1 IV], BP95 [lin-39(n1760) III; jcIs1 IV], BP121 [lin-39(n1760)III; zzEx10], BP123 leff-1(hy21) II; unc-36(e251), lin-39(n1490) III; zzEx10], BP124 [unc-36(e251), lin-39(n1490) III; zzEx10], and BP126 [ceh-20(ay42), unc36(e251)III; zzEx10].

BP92 was constructed by mating jcIs1 males with unc-36(e251), lin39 (n1490)III hermaphrodites at $15^{\circ} \mathrm{C}(10 \%$ of the hermaphrodites have normal vulva at $15^{\circ} \mathrm{C}$ ) and using outcross males for mating with eff1(hy21) hermaphrodites. Rol F1s of this mating were isolated, and F2s were screened for Unc; Vul;Rol worms. These worms were further isolated and F3 were screened for Eff; Unc;Vul;Rol that showed temperaturesensitivity regarding the Vul phenotype. BP93 was constructed during construction of BP92, only Unc;Vul;Rol non-Eff worms were isolated from F3 progeny. BP94 was constructed by mating jcIs1 males with BP76 [eff-1(hy21)II;jcIs1 IV] and using outcross males for mating with MT4009 [1in-39(n1760)/dpy-17(e164);unc-32(e189)III] hermaphrodites. After isolation of Rol F1s, F2s were screened for Rol;Vul worms, and the progeny of these worms (F3) were screened for Eff; Vul;Rol mutants. BP95 was constructed during construction of BP94, only Vul;Rol non-Eff worms were isolated from F3 progeny. BP121 was constructed by mating him-8(e1489)IV males with FC50 [zzEx10(p/E3, pRF4)] hermaphrodites and using outcross males for mating with MT4009 [1in-39(n1760)/dpy17(e164);unc-32(e189)III] hermaphrodites. Rol F1s that express eff$1 p:: G F P$ were isolated, and Rol F2s were cloned. F3s were screened for Vul eff-1p::GFP expressing mutants. BP123 was constructed by mating FC50 [zzEx10(pJE3, pRF4)] males with BP92 [eff-1(hy21)II;unc36(e251);in-39(n1490ts)III; jcIs1] hermaphrodites at $15^{\circ} \mathrm{C}$. Rol non-Unc F1s were transferred to the restrictive temperature of $20^{\circ} \mathrm{C}$, and Eff F2s that express eff-1p::GFP in the epithelia were isolated. From these worms, F3 were screened for $\mathrm{Eff}_{;} \mathrm{Unc} ; \mathrm{Vul}$ worms that express eff$1 p:: G F P$. BP124 was constructed during construction of BP123, only Vul F2s expressing eff-1p::GFP were isolated and screened for the absence of Eff progeny. BP126 was constructed by mating FC50 [zzEx10(p/E3, pRF4)] males with NH2296 [ceh-20(ay42) unc-36(e251)/sma-3(e491) unc-36(e251) III] hermaphrodites. Rol non-Unc F1s were isolated, and F2 were scored for Unc; Vul worms that express eff-1p:: GFP.
Immunofluorescence and microscopy

To image larvae, GFP-expressing worms were anesthetized with $5 \mathrm{mM}$ $\mathrm{NaN} 3$ or $0.01 \%$ levamisole or fixed briefly in $2 \%$ paraformaldehyde. To check aim-1::GFP expression, we fixed and stained worms using the mouse monoclonal antibody $\mathrm{MH} 27$ (1:300 dilution) that recognizes AJM-1 within the adherens junctions (zonula adherens) of epithelial cells (Francis and Waterston 1991; Shemer et al. 2000). Worms were staged and visualized as described (Shemer et al. 2000). To follow proliferation of cells, worms were analyzed by Nomarski optics.

\section{Acknowledgments}

We thank T. Stiernagle of the Caenorhabditis Genetics Center for strains; E. Lifschitz, P. Sternberg, B.-Z. Shilo, and Z. Paroush for critical reading of the manuscript; and $M$. Glickman, R. Reshef, and B. Horwitz for valuable comments. G.S. was supported by a pre-doctoral fellowship from the Landau Fund of Mifal Hapais. This research was supported by the Israel Science Foundation-The Charles H. Revson Foundation (grant 203/00-2), Binational Science Foundation and Human Frontier Science Program.

The publication costs of this article were defrayed in part by payment of page charges. This article must therefore be hereby marked "advertisement" in accordance with 18 USC section 1734 solely to indicate this fact.

\section{References}

Alper, S. and Kenyon, C. 2001. REF-1, a protein with two bHLH domains, alters the pattern of cell fusion in C. elegans by regulating Hox protein activity. Development 128: 1793-1804.

. 2002. The zinc finger protein REF-2 functions with the Hox genes to inhibit cell fusion in the ventral epidermis of C. elegans. Development 129: 3335-3348.

Amthor, H., Christ, B., Weil, M., and Patel, K. 1998. The importance of timing differentiation during limb muscle development. Curr. Biol. 8: 642-652.

Brenner, S. 1974. The genetics of Caenorhabditis elegans. Genetics 77: 71-94.

Ch'ng, Q. and Kenyon, C. 1999. egl-27 generates anteroposterior patterns of cell fusion in $C$. elegans by regulating Hox gene expression and Hox protein function. Development 126: 3303-3312.

Clandinin, T.R., Katz, W.S., and Sternberg, P.W. 1997. Caenorhabditis elegans Hom-C genes regulate the response of vulval precursor cells to inductive signal. Dev. Biol. 182: 150-161.

Clark, S.G., Chisholm, A.D., and Horvitz, H.R. 1993. Control of cell fates in the central body region of C. elegans by the homeobox gene lin-39. Cell 74: 43-55.

Eisenmann, D.M., Maloof, J.N., Simske, J.S., Kenyon, C., and Kim, S.K 1998. The $\beta$-catenin homolog BAR-1 and LET-60 ras coordinately regulate the hox gene lin-39 during Caenorhabditis elegans vulval development. Development 125: 3667-3680.

Eizinger, A. and Sommer, R.J. 1997. The homeotic gene lin-39 and the evolution of nematode epidermal cell fates. Science 278: 452-454.

Felix, M.-A. 1999. Evolution of developmental mechanisms in nematodes. J. Exp. Zool. 285: 3-18.

Francis, G.R. and Waterston, R.H. 1991. Muscle cell attachment in Caenorhabditis elegans. J. Cell Biol. 114: 465-479.

Gleason, J.E., Korswagen, H.C., and Eisenmann, D.M. 2002. Activation of Wnt signaling bypasses the requirement for RTK/Ras signaling during C. elegans vulval induction. Genes \& Dev. 16: 1281-1290.

Graba, Y., Aragnol, D., and Pradel, J. 1997. Drosophila Hox complex downstream targets and the function of homeotic genes. Bioessays 19: $379-388$.

Grandien, K. and Sommer, R.J. 2001. Functional comparison of the nematode Hox gene lin-39 in C. elegans and P. pacificus reveals evolutionary conservation of protein function despite divergence of primary sequences. Genes \& Dev. 15: 2161-2172.

Grant, K., Hanna-Rose, W., and Han, M. 2000. sem-4 promotes vulval cell-fate determination in Caenorhabidtis elegans through regulation of lin-39 Hox. Dev. Biol. 224: 496-506.

Harris, W.A. and Hartenstein, V. 1991. Neuronal determination without cell division in Xenopus embryos. Neuron 6: 499-515.

Hoier, E.F., Mohler, W.A., Kim, S.K., and Hajnal, A. 2000. The Caenorha- 
bidtis elegans APC-related gene apr-1 is required for epithelial cell migration and Hox gene expression. Genes \& Dev. 14: 874-886.

Koeppen, M., Simske, J.S., Sims, P.A., Firestein, B.L., Hall, D.H., Radice, A.D., Rongo, C., and Hardin, J.D. 2001. Cooperative regulation of AJM-1 controls junctional integrity in Caenorhabditis elegans epithelia. Nat. Cell Biol. 3: 983-991.

Kornfeld, K. 1997. Vulval development in Caenorhabditis elegans. Trends Genet. 13: 55-61.

Liu, J. and Fire, A. 2000. Overlapping roles of two Hox genes and the exd ortholog ceh-20 in diversification of the C. elegans postembryonic mesoderm. Development 127: 5179-5190.

Louvet-Vallee, S., Kolotuev, I., Podbilewicz, B., and Felix, M.-A. 2002. Control of vulval competence and centering in the nematode Oscheius sp.1 CEW1. Genetics (in press).

Maloof, J.N. and Kenyon, C. 1998. The Hox gene lin-39 is required during C. elegans vulval induction to select the outcome of Ras signaling. Development 125: 181-190.

Mann, R.S. and Affolter, M. 1998. Hox proteins meet more partners. Curr. Opin. Gen. \& Dev. 8: 423-429.

Mohler, W.A., Shemer, G., del Campo, J., Valansi, C., Opoku-Serebuoh, E., Scranton, V., Assaf, N., White, J.G., and Podbilewicz, B. 2002. The type I membrane protein EFF-1 is essential for developmental cell fusion in C. elegans. Dev. Cell 2: 355-362.

Podbilewicz, B. and White, J.G. 1994. Cell fusions in the developing epithelia of C. elegans. Dev. Biol. 161: 408-424.

Riddle, D., Blumenthal, T., Meyer, B., and Priess, J. 1997. C. elegans II. pp. 902-1047. Cold Spring Harbor Laboratory Press, Cold Spring Harbor, NY.

Salser, S.J. and Kenyon, C. 1996. A C. elegans Hox gene switches on, off, on and off again to regulate proliferation, differentiation and morphogenesis. Development 122: 1651-1661.

Sharma-Kishore, R., White, J.G., Southgate, E., and Podbilewicz, B. 1999. Formation of the vulva in C. elegans: a paradigm for organogenesis. Development 126: 691-699.

Shemer, G. and Podbilewicz, B. 2000. Fusomorphogenesis: Cell fusion in organ formation. Dev. Dyn. 218: 30-51.

Shemer, G., Kishore, R., and Podbilewicz, B. 2000. Ring formation drives invagination of the vulva in C. elegans: Ras, cell fusion and cell migration determine structural fates. Dev. Biol. 221: 233-248.

Wang, B.B., Muller-Immergluck, M.M., Austin, J., Robinson, N.T., Chisholm, A., and Kenyon, C. 1993. A homeotic gene cluster patterns the anteroposterior body axis of C. elegans. Cell 74: 29-42.

Witze, E. and Rothman, J.E. 2002. Cell fusion: An EFFicient sculptor. Curr. Biol. 12: R467-R469.

Ying, Q.L., Nichols, J., Evans, E.P., and Smith, A.G. 2002. Changing potency by spontaneous fusion. Nature 416: 545-548. 


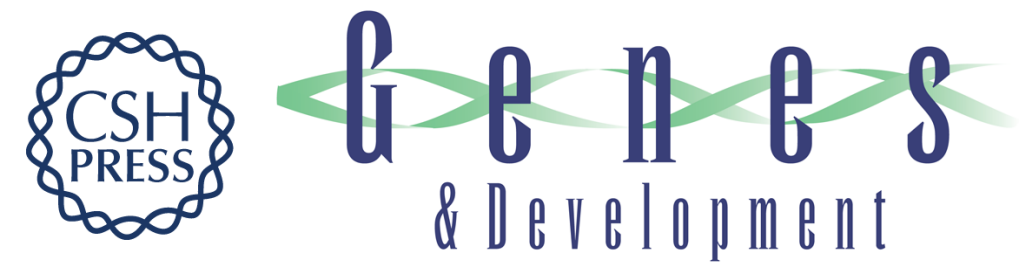

\section{LIN-39/Hox triggers cell division and represses EFF-1/fusogen-dependent vulval cell fusion}

Gidi Shemer and Benjamin Podbilewicz

Genes Dev. 2002, 16:

Access the most recent version at doi:10.1101/gad.251202

Supplemental
Material http://genesdev.cshlp.org/content/suppl/2002/12/22/16.24.3136.DC1

References This article cites 31 articles, 14 of which can be accessed free at:

http://genesdev.cshlp.org/content/16/24/3136.full.html\#ref-list-1

License

Email Alerting Receive free email alerts when new articles cite this article - sign up in the box at the top

Service right corner of the article or click here.

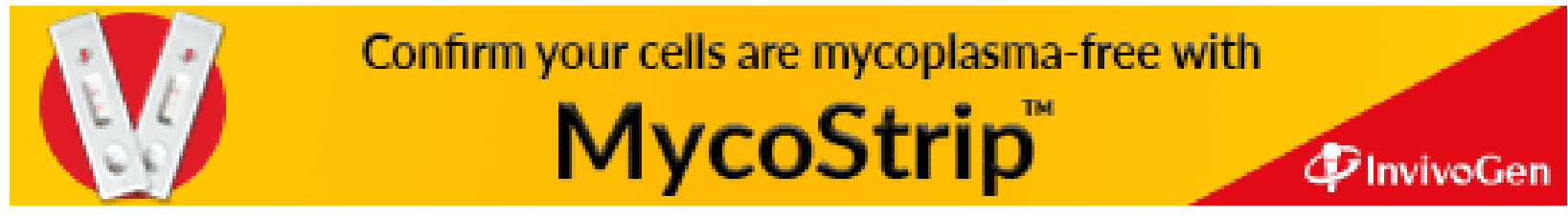

